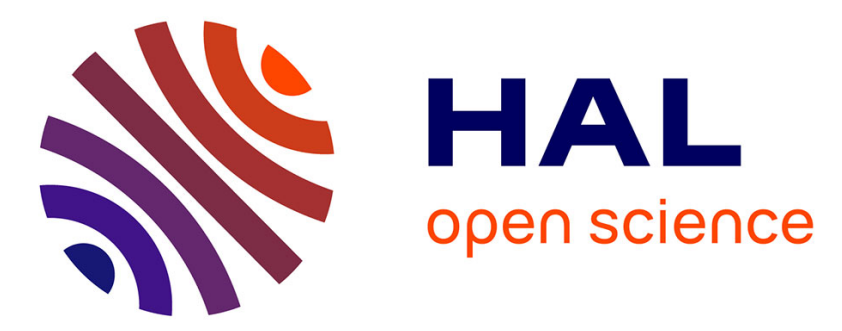

\title{
Detection of Bartonella spp. in Cimex lectularius by MALDI-TOF MS
}

\author{
Basma El Hamzaoui, Maureen Laroche, Philippe Parola
}

\section{To cite this version:}

Basma El Hamzaoui, Maureen Laroche, Philippe Parola. Detection of Bartonella spp. in Cimex lectularius by MALDI-TOF MS. Comparative Immunology, Microbiology and Infectious Diseases, 2019, 64, pp.130-137. 10.1016/j.cimid.2019.03.001 . hal-02466849

\section{HAL Id: hal-02466849 \\ https://hal-amu.archives-ouvertes.fr/hal-02466849}

Submitted on 22 Oct 2021

HAL is a multi-disciplinary open access archive for the deposit and dissemination of scientific research documents, whether they are published or not. The documents may come from teaching and research institutions in France or abroad, or from public or private research centers.
L'archive ouverte pluridisciplinaire HAL, est destinée au dépôt et à la diffusion de documents scientifiques de niveau recherche, publiés ou non, émanant des établissements d'enseignement et de recherche français ou étrangers, des laboratoires publics ou privés.

\section{다(1) $\$$}

Distributed under a Creative Commons Attribution - NonCommerciall 4.0 International 
1 Detection of Bartonella spp. in Cimex lectularius by MALDI-TOF MS

2 Basma El Hamzaoui ${ }^{1,2}$, Maureen Laroche ${ }^{1,2}$, Philippe Parola ${ }^{1,2^{*}}$

$3 \quad{ }^{1}$ Aix Marseille Univ, IRD, AP-HM, SSA, VITROME, Marseille, France

$4 \quad{ }^{2}$ Institut Hospitalo-Universitaire Méditerranée Infection, Marseille, France

$5 \quad{ }^{*}$ Corresponding author: Prof. Philippe Parola. VITROME, Institut Hospitalo-Universitaire

6 Méditerranée Infection. 19-21 Boulevard Jean Moulin 13005 Marseille France Phone: + 33

7 (0) 4137324 01. Fax: + 33 (0) 4137324 02. Email address: philippe.parola@ univ-amu.fr

8

9 KEYWORDS: Cimex lectularius, Bartonella quintana, Bartonella henselae, MALDI-TOF

10 MS. 


\section{Abstract:}

13 Bed bugs are small hematophagous insects. They are found in temperate and tropical climates 14 around the world. Their vectorial capacity for several pathogens, including Bartonella spp., has been suspected. An experimental study of artificial infection of Cimex lectularius with Bartonella quintana and Bartonella henselae bacteria was developed to evaluate the ability of

17 MALDI-TOF MS to simultaneously identify bed bugs and their infectious status. This experimental study confirmed the ability of MALDI-TOF MS to identify bed bugs. In addition, it was able to differentiate between control bed bugs, bed bugs infected with Bartonella quintana and bed bugs infected with Bartonella henselae, with an identification

21 percentage above $90 \%$. 


\section{Introduction}

Bed bugs are brown, flat parasitic arthropods belonging to the Cimicidae family. Males and females are strictly hematophagous and can live for 12 months without feeding [1]. The two cosmopolite species, Cimex lectularius and Cimex hemipterus, are most often found among humans [2]. Because of their hematophagous habits and intimate association with humans, bed bugs have long been suspected in disease transmission, but their vectorial capacity has rarely been studied [1].

Recently, bed bugs have increased significantly in frequency and in geographical distribution. An increasing number of infestations have been reported in Europe and America, causing several clinical and psychological problems $[1,3,4]$.

Bartonella spp. are fastidious, aerobic, Gram-negative bacteria. Bartonella quintana is the agent of trench fever, known to be transmitted by the human body louse (Pediculus humanus humanus) [5]. Bartonella henselae is the agent of cat-scratch disease, shown to be transmitted by fleas [6]. Recently, B. quintana DNA was detected in C. hemipterus (tropical bed bugs) in Rwanda [7]. Subsequently, C. lectularius was shown to be a potential vector of this bacterium using an experimental study of C. lectularius bed bug infection with B. quintana [5].

The monitoring and identification of bed bugs, as well as the detection of associated pathogens, are crucial steps for the surveillance of diseases potentially transmitted by these arthropods. Currently, bed bugs are identified primarily by observing morphological characteristics. However, the number of entomologists specialized in taxonomy is very small, and the distinction between $C$. lectularius and $C$. hemipterus cannot be made by a nonspecialist [8]. As an alternative, molecular tools have been used to improve identification and compensate for the limitations of morphological identification [9].

In recent years, matrix-assisted laser desorption/ionization time-of-flight mass spectrometry (MALDI-TOF MS) has emerged as an efficient tool for arthropod identification [9] in 
mosquitoes [10-12], fleas [13] and ticks [14]. More recently, MALDI-TOF MS has been successfully applied to differentiate ticks infected or not with Borrelia crocidurae [15] or Rickettsia spp. [16], fleas infected or not with B. quintana and B. henselae [17] and mosquitoes infected or not with Plasmodium berghei [18].

In this work, we set up an experimental study of bed bug infection with $B$. quintana and $B$. henselae. We ranked the bed bug groups according to their infectious status through molecular methods, and we evaluated the ability of MALDI-TOF to differentiate the different groups and different pathogens.

\section{Materials and methods}

Ethical statement

Human blood was obtained from the "Etablissement Français du Sang" (EFS, French Blood Establishment) accredited by the Institutional Animal Care of IHU Méditerranée Infection.

\section{Bed bug rearing}

Bed bugs (C. lectularius) obtained from the Cimex store (London, England, cimexstore.co.uk) were maintained under standard laboratory rearing conditions, as previously described [5]. The breeding containers were placed in the laboratory incubator at $60 \%$ humidity and $22^{\circ} \mathrm{C}$, and the same conditions were used for adults and larvae [5]. The bed bugs were fed once a week using a Hemotek artificial feeder machine (Hemotek 5W1; Discovery Workshops, Accrington, UK). The device was covered by an artificial membrane of Parafilm M (SigmaAldrich, Saint-Louis, Missouri, USA) and filled with $2 \mathrm{~mL}$ of blood [19].

\section{Bartonella quintana and Bartonella henselae strain}

All experiments with $B$. quintana and $B$. henselae were conducted inside a biosafety cabinet in a Level 2 Biosafety Room (BSL2). The two bacterial strains (strain B. quintana Oklahoma 
ATCC 49793, strain B. henselae Marseille) were cultured on Columbia sheep blood agar plates (5\%, BioMérieux, Marcy l'Etoile, France) at $37^{\circ} \mathrm{C}$ in an atmosphere enriched with $5 \%$ $\mathrm{CO} 2$ [13]. After 10 days of culture, the bacteria were collected and transferred into a tube containing $400 \mu \mathrm{L}$ of phosphate buffered saline (PBS), pH 7.2 (PBS, BioMérieux, Marcy l'Etoile, France). Two hundred microliters of each bacterial suspension mixed with two $\mathrm{mL}$ of human blood were used for artificial infection of bed bugs. An aliquot of two mL of human whole blood containing $200 \mu \mathrm{L}$ of PBS was used to feed the control group $[14,17]$.

Spectra from both strains were also obtained using MALDI-TOF MS. One colony was directly deposited on a MALDI-TOF target plate (Bruker Daltonik), and 4 spots were made for each bacterium. Each spot was covered with $2 \mu \mathrm{L}$ of matrix solution (saturated solution of a-cyano-4-hydroxycinnamic acid in $50 \%$ acetonitrile, and $2.5 \%$ trifluoracetic-acid) and the matrix-sample was crystallized by air-drying at room temperature for 5 minutes, as previously described (X). Measurements were performed with an Autoflex II mass spectrometer (Bruker Daltonik) equipped with a 337-nm nitrogen laser. Spectra were recorded in the positive linear mode (delay, $170 \mathrm{~ns}$; ion source 1 voltage, $20 \mathrm{kV}$; ion source 2 voltage, $18.5 \mathrm{kV}$; lens voltage, $7 \mathrm{kV}$; mass range, 2-20 kDa) [20].

\section{Artificial infection of bed bugs}

Three groups were created, including a group containing $100 \mathrm{C}$. lectularius exposed to $B$. quintana, a group containing $100 C$. lectularius exposed to B. henselae and a control group containing 50 C. lectularius.

Each bed bug group was fed with three successive human blood meals (every 48 hours). Control bed bugs were fed with Bartonella-free blood mixed with $200 \mu \mathrm{L}$ of PBS and exposed bed bugs were fed with two $\mathrm{mL}$ of blood infected with $200 \mu \mathrm{L}$ of bacterial suspension of B. henselae and B. quintana [5,17] 
Two hundred microliters of infected blood were tested using qPCR, to ensure the presence of B. quintana and B. henselae. One hundred microliters of each inoculum were cultured on sheep blood agar to ensure bacterial viability and, at the same time, calculate the concentration by making a serial dilution of culture from $10^{-1}$ to $10^{10}[5,21]$.

\section{DNA extraction and qPCR detection of $B$. quintana and $B$. henselae in bed bugs}

From the third day post infection, 10 bed bugs were collected every 48 hours in each group. Each bed bug was washed with $70 \%$ ethanol, rinsed twice with distilled water and dried with paper. Half of the abdomen was reserved for the detection of B. quintana and B. henselae by qPCR and the head was stored at $-20^{\circ} \mathrm{C}$ for MALDI-TOF MS tests $[5,17]$.

The bed bug samples reserved for molecular biology were incubated overnight at $56^{\circ} \mathrm{C}$ in 180 $\mu \mathrm{L}$ of G2 buffer (Qiagen, Hilden, Germany) (30 mM Tris-Cl, 30 mMEDTA; 5\% Tween 20, 0.5\% Triton X-100; $800 \mathrm{mMGuHCl}$ ) and $20 \mu \mathrm{L}$ of proteinase K (Qiagen, Hilden, Germany) (activity of $600 \mathrm{mAU} / \mathrm{mL}$ solution or $40 \mathrm{mAU} / \mathrm{mg}$ of protein). DNA extraction was performed in the automatic extractor EZ1 (Qiagen, Hilden, Germany). The presence of B. quintana and B. henselae DNA in these bed bugs was assessed by B. quintana and B. henselae-specific real-time PCRs targeting the yopP3 gene [22] and pap31 gene [23], respectively. The presence of Bartonella spp. was tested individually in each bed bug.

\section{Detection of Bartonella spp. in Cimex lectularius by MALDI-TOF MS}

Samples reserved for MALDI-TOF MS (bed bug head) were washed a second time with HPLC-grade water and acetonitrile (Fluka, Buchs, Switzerland). They were then crushed using the automated grinding device TissueLyser (Qiagen, Hilden, Germany) 3 times for 90 seconds at a frequency of $30 \mathrm{Hertz}$, with a pinch of glass powder (Sigma, Lyon, France) and a mix of $20 \mu \mathrm{L}$ of $70 \%$ formic acid (v/v) and $20 \mu \mathrm{L}$ of $50 \%$ acetonitrile (v/v) (Fluka, Buchs, Switzerland). 
After centrifugation at $200 \mathrm{~g}$ for 1minute, $1 \mu \mathrm{L}$ of supernatant of each sample was deposited in quadruplicate on the MALDI-TOF plate and covered by $1 \mu \mathrm{L}$ of CHCA matrix solution composed of saturated $\alpha$-cyano-4-hydroxycynnamic acid (CHCA) (Sigma, Lyon, France), $50 \%$ (v/v) acetonitrile (Fluka, Buchs, Switzerland), 2.5\% trifluoroacetic acid (v/v) (Aldrich, Dorset, UK) and HPLC-grade water. The target was then placed in the Microflex LT 171 MALDI-TOF mass spectrometer (Bruker Daltonics) for analysis [9,17] (Laroche et al., personal communication).

\section{MALDI-TOF MS parameters}

Protein mass profiles were generated using a Microflex LT MALDI-TOF (Bruker Daltonics, Germany) mass spectrometer, with detection in positive ion linear mode at a laser frequency of $50 \mathrm{~Hz}$ in a mass range $2-20 \mathrm{kDa}$. The acceleration voltage was set to $20 \mathrm{kV}$, and the time of extraction delay was $200 \mathrm{~ns}$. Each spectrum corresponds to the ions obtained from 240 laser shots fired in six regions of the same deposit on the ground plate and acquired automatically using the function of the Flex Control AutoXecute V.2.4 software (Bruker Daltonics).

The spectra were visualized with Flex analysis v.3.3 software and exported to ClinProTools2.2 and MALDI Biotyper v.3.0 (Bruker Daltonics, Germany) for data processing. To obtain usable results, we evaluated the reproducibility of each group using the ClinProTools 2.2 software.

Bed bug spectra were chosen based on their reproducibility and intensity for all MS analyses, including database creation. Our database was upgraded with spectra of 4 to 11 specimens of non-infected bed bugs or bed bugs infected with B. quintana or B. henselae.

\section{Blind tests}

Blind test analysis was used to evaluate the ability of MALDI-TOF MS to distinguish the three groups. After the evaluation of the quality and the reproducibility of the spectra, a dendrogram was established for each group to evaluate the homogeneity and a high quality 
spectra within each cluster, and high quality and reproducible MALDI-TOF MS spectra from the head of $4 C$. lectularius controls, $5 C$. lectularius infected with $B$. henselae and $11 C$. lectularius infected with $B$. quintana were introduced into our in-house arthropod database (available on request) (Species of this database are reported in Supplementary data 1).

Then, a total of 34 spectra of $C$. lectularius controls, 61 spectra of $C$. lectularius infected with B. henselae and 62 spectra of $C$. lectularius infected with B. quintana were queried against the upgraded database.

\section{MALDI-TOF MS biomarker mass set}

To evaluate the reproducibility of the MS profiles, the spectra of the three groups (bed bugs infected with $B$. quintana, bed bugs infected with $B$. henselae and bed bug controls) were imported into the ClinProTools 2.2 software. For the detection of the discriminant peaks associated with infection status, we compared the average spectrum of the three categories of bed bugs. The software was used to generate a peak list for each group in the mass range of 2 to $20 \mathrm{kDa}$ and to identify the discriminant peaks. The parameters of the ClinProTools 2.2 software for the generation of the spectra were as follows: resolution of 800 ; noise threshold of 2.50; maximum peak of $800 \mathrm{ppm}$ and correspondence with calibration peaks of $20 \%$. For the calculation of the peak, the peak was made on single spectra, with a signal-to-noise threshold of 2.50 and an aggregation of $800 \mathrm{ppm}$. The spectra were then analyzed with the genetic algorithm (GA) model, which provided a list of discriminant peaks. Manual inspection and peak validation by the operator gave a "recognition capability" (RC) value with the highest "cross-validation" (CV) value.

\section{Results}

\section{Acquisition of Bartonella quintana and Bartonella henselae by bed bugs}


The inoculum used for artificial infection with B. quintana contained $3 \times 10^{8} \mathrm{CFU} / \mathrm{mL}$ bacteria and the one used for artificial infection with $B$. henselae contained $2 \times 10^{7} \mathrm{CFU} / \mathrm{mL}$ bacteria.

Three days after the last infective meal, ten bed bugs from each group were subsequently collected every two days. At the end of the collections, we had a total of 80 bed bugs exposed to $B$. quintana, 80 bed bugs exposed to B. henselae and 40 bed bug controls.

Quantitative PCR results demonstrated the presence of B. quintana in all exposed bed bugs, with cycle threshold $(\mathrm{Ct})$ values ranging from 16.19 to 27.69 , and the presence of $B$. henselae in all exposed bed bugs (100\% infection rate), with cycle threshold $(\mathrm{Ct})$ values ranging from 19.13 to 24.46. All control fleas tested negative.

\section{MALDI-TOF MS for detection of $B$. quintana and $B$. henselae in bed bugs}

The spectra sorted by Flex Control AutoXecute V.2.4 software were analyzed by ClinProTools 2.2 software to check the reproducibility of each category (Figure 1). This analysis showed the presence of some peaks only in bed bugs infected with B. quintana and other peaks present only in bed bugs infected with $B$. henselae but absent in controls (Figure 2).

The ClinProTools 2.2 software also calculated and created the principal component analysis (PCA) of the three groups of bed bugs. It compared the group of infected bed bugs with $B$. quintana and the control group (Figure 3.A), as well as the group of infected bed bugs with $B$. henselae with the control group (Figure 3.B).

To better identify discriminating peaks between the infected bed bugs and the control bed bugs, we used the genetic algorithm tool of the ClinProTools 2.2 software. We compared the spectra of 62 bed bugs infected with $B$. quintana and 34 spectra of control bed bugs. Similarly with the second pathogen, we compared 61 specimens of infected bed bugs with $B$. henselae and 34 control bed bugs. 
193

194

195

196

197

198

199

200

201

202

203

204

205

206

207

208

209

210

211

212

213

214

215

216

217

The genetic algorithm model and operator peak picking generated 10 discriminating peaks between bed bug controls and bed bugs infected with $B$. henselae (Table 1), with a recognition capability $(\mathrm{RC})$ and cross-validation $(\mathrm{CV})$ of $100 \%$ for both, and nine discriminating peaks between bed bug controls and bed bugs infected with B. quintana (Table 2), with a recognition capability (RC) of $100 \%$ and cross-validation (CV) of $98.53 \%$ (Figure $4,5)$.

The last step of the MALDI TOF analysis was the blind test of the spectra of bed bugs against our arthropod database, previously upgraded with the reference spectra of each bed bug category (infected with $B$. quintana, infected with $B$. henselae, and controls). Bed bug species identification was correct for all reproducible spectra. Regarding the infection status, identification was correct for 56/62 C. lectularius infected with B. quintana (90.32\%), but 6 of these 62 B. quintana-infected bed bugs were identified either as C. lectularius controls $(\mathrm{n}=3)$ or C. lectularius infected with Borrelia recurrentis $(\mathrm{n}=3)$.

The identification was correct for 55/61 C. lectularius infected with B. henselae $(90.16 \%$ ), but 6 of these $61 \mathrm{~B}$. henselae-infected bed bugs were identified either as C. lectularius controls (n=4) or C. lectularius infected with B. quintana $(\mathrm{n}=2)$. Finally, the identification was correct for $31 / 34$ C. lectularius controls, but 3 of these 34 C. lectularius controls were identified either as C. lectularius infected with B. quintana $(\mathrm{n}=2)$ or $C$. lectularius infected with $B$. recurrentis $(\mathrm{n}=1)$ (Table 3$)$.

\section{Discussion}

This work presents a preliminary study assessing the ability of MALDI-TOF MS to differentiate between non-infected bedbugs and bedbugs infected with two different species of Bartonella using a previously validated study of artificial infection of C. lectularius with Bartonella spp.[5]. The protocol used was developed by comparing the reproducibility and 
quality of spectra obtained from different laboratory bed bug body parts. It was later validated on field bed bugs from very different geographical areas (urban area in south of France, rural

220

221 village in Senegal) (Laroche et al., personal communication). It proved its effectiveness on infected bed bugs and controls with $88.5 \%$ of all the spectra analyzed included in the study, based on quality and reproducibility. These results are encouraging, since these spectra allowed an identification rate of bed bugs infected by B. quintana and B. henselae above $90 \%$. In the case of slight heterogeneity within the same group, the more reference spectra one has, the more accurate the blind test analyses become. For this proof of concept step, it is necessary to keep only the best spectra to assess the performance of the tool in a blind test analysis. A validated database following a successful blind test analysis with high quality spectra means that each of these spectra can be used eventually as a reference to encompass all the future diversity encountered.

Two groups of bed bugs were fed blood infected with B. henselae or B. quintana separately; the qPCR showed the acquisition of both bacteria by bed bugs with a $100 \%$ infection rate, in accordance with what was previously reported [5].

After its success in microbiology, MALDI-TOF MS has emerged in the field of entomology and identification of arthropods. To increase the efficiency and shorten the time-to-result of MALDI-TOF MS analyses, it is necessary to standardize the sample preparation protocol, which also makes it possible to reproduce the tests in different research laboratories $[8,16]$. This standardization encompasses several parameters; the arthropod body part used, sample washing steps and lastly, speed and frequency of grinding. These parameters vary according to the characteristics of each arthropod, requiring the development of a sample preparation protocol for MALDI-TOF MS specific to each arthropod [10,24].

During this work, we followed the standardized protocol implemented in our laboratory for bed bug analyses (Laroche et al., personal communication). This protocol has proven its 

$(88.5 \%)$ on all spectra analyzed.

245 In this study, qPCR allowed proper classification of the three groups of bedbugs according to their infectious status. As demonstrated in previous work, bed bug heads provide reproducible spectra. Therefore, 50 specimens only were selected as controls. The database was upgraded with reference spectra of each category. The blind test showed a percentage of identification ranging from $90.16 \%$ to $91 \%$ for all three groups.

Regarding the identification of incorrect Bartonella species, we have reported in previous studies that the proteins of the infected arthropod itself could play a role in the differentiation of the two groups (infected/non-infected). Indeed, in Plasmodium-infected mosquitoes, a lower number of peaks and a decreased intensity of others was found compared to control mosquitoes. The role of down-regulated mosquito proteins, involved in the immune response, can be hypothesized [18]. In the mass spectrometry approach used in this study, we could not assess the exact origin of each peak. We can hypothesize that the immune response may play a role in the identification of Bartonella spp. in bed bugs as well. Assuming that the proteins regulated are the same for the two bacteria, we would have common peaks associated with bed bug proteins, and specific ones for each bacterium.

In a previous work, we evaluated the ability of MALDI-TOF MS to distinguish between fleas infected or not by $B$. quintana and $B$. henselae, and then we noticed the presence of a third group, the group of fleas exposed to the bacteria, but negative in qPCR. This category was well-differentiated from the others by MALDI-TOF MS. It had been suggested that the separation of an exposed group might be explained by the presence of the proteins of the immune response [17]. In the current study, we did not have an exposed uninfected group identified by MALDI-TOF, since the rate of infection was $100 \%$. The few errors that have been noticed regarding the status of infection might be also linked with the immune response 
in bed bugs, where the immune response is more important in some samples or weak or even absent in others. The listed discriminating peaks are those which allow distinction of the two groups with the lowest number of peaks. Several other peaks are actually discriminative

27 between the two groups and are not reported. This can explain why no common

272 discriminating peaks are found in the list presented in this study.

273 Compared to other arthropod spectra, bed bug samples demonstrate significant intra-group heterogeneity. It is therefore impossible to have a relevant identification with 2 or 3 reference spectra, as was the case for highly reproducible Australian mosquito spectra, for example [25]. An upgrade of the database with a high number of bed bug reference spectra should be a satisfactory approach to avoid misidentifications.

278 In conclusion, after a preliminary work on fleas and the present study on bed bugs, MALDI-

279 TOF MS appears to be a promising tool for differentiating arthropods infected or not with Bartonella, and further complementary studies are needed to better characterize the

281 differential proteome of infected and non-infected arthropods. Perspectives include testing this tool in the field during entomological studies. Since the MALDI-TOF MS device is bought for microbiology perspectives, its use in entomology does not require additional cost and might be very useful, given the resurgence of bed bugs.

\section{Tables}

\begin{tabular}{|c|c|c|}
\hline \multicolumn{3}{|c|}{$\begin{array}{c}\text { Comparison between Cimex lectularius } \\
\text { infected with Bartonella henselae and } \\
\text { controls }\end{array}$} \\
\hline $\begin{array}{c}\text { Number } \\
\text { of } \\
\text { peaks }\end{array}$ & $\begin{array}{c}\text { Controls } \\
\text { Mass (m/z) } \\
(\mathrm{Da})\end{array}$ & $\begin{array}{c}\text { Infected } \\
\text { Mass (m/z) } \\
(\mathrm{Da})\end{array}$ \\
\hline 1 & Absent & $\mathbf{4 5 6 7}$ \\
\hline 2 & Absent & $\mathbf{5 5 1 3}$ \\
\hline 3 & $\mathbf{5 9 9 9}$ & Absent \\
\hline 4 & Absent & $\mathbf{7 3 3 2}$ \\
\hline
\end{tabular}




\begin{tabular}{|c|c|c|}
5 & $\mathbf{7 4 9 1}$ & Absent \\
\hline 6 & $\mathbf{7 7 4 2}$ & Absent \\
\hline 7 & Absent & $\mathbf{9 0 2 5}$ \\
\hline 8 & $\mathbf{1 0 0 6 2}$ & Absent \\
\hline 9 & $\mathbf{1 1 9 8 9}$ & Absent \\
\hline 10 & $\mathbf{1 4 9 8 2}$ & Decreased \\
\hline
\end{tabular}

Table 1: Discriminant peaks between bed bug controls and bed bugs infected with Bartonella

\begin{tabular}{|c|c|c|}
\hline \multicolumn{3}{|c|}{$\begin{array}{c}\text { Comparison between Cimex lectularius } \\
\text { infected with Bartonella quintana and } \\
\text { controls }\end{array}$} \\
\hline $\begin{array}{c}\text { Number } \\
\text { of } \\
\text { peaks }\end{array}$ & $\begin{array}{c}\text { Controls } \\
\text { Mass (m/z) } \\
\text { (Da) }\end{array}$ & $\begin{array}{c}\text { Infected } \\
\text { Mass (m/z) } \\
(\mathrm{Da})\end{array}$ \\
\hline 1 & Absent & $\mathbf{3 9 6 7}$ \\
\hline 2 & Absent & $\mathbf{4 5 7 4}$ \\
\hline 3 & Absent & $\mathbf{4 8 6 3}$ \\
\hline 4 & $\mathbf{5 1 3 9}$ & Absent \\
\hline 5 & $\mathbf{5 9 9 7}$ & Absent \\
\hline 6 & $\mathbf{6 5 3 9}$ & Absent \\
\hline 7 & Absent & $\mathbf{7 2 9 7}$ \\
\hline 8 & Absent & $\mathbf{1 1 1 3 2}$ \\
\hline 9 & Absent & $\mathbf{1 4 5 9 2}$ \\
\hline
\end{tabular}

Table 2: Discriminant peaks between bed bug controls and bed bugs infected with Bartonella quintana detected by the genetic algorithm model analyzed with ClinProTools software. 


\begin{tabular}{|c|c|c|c|l|}
\hline Categories & Total & Spectra & MS Good \\
& sollected & spectra & introduced in & identification \\
the database & & 4 & $\mathbf{3 1 / 3 4}(\mathbf{9 1 \%})$ \\
\hline $\begin{array}{c}\text { Cimex lectularius } \\
\text { controls }\end{array}$ & 40 & 38 & 5 & $\mathbf{5 5 / 6 1}(\mathbf{9 0 , 1 6 \% )})$ \\
\hline $\begin{array}{c}\text { Cimex lectularius } \\
\text { infected with Bartonella } \\
\text { henselae }\end{array}$ & 80 & 66 & 11 & $\mathbf{5 6 / 6 2}(\mathbf{9 0 ,}, \mathbf{3 2 \%})$ \\
\hline $\begin{array}{c}\text { Cimex lectularius } \\
\text { infected with Bartonella } \\
\text { quintana }\end{array}$ & 80 & 73 & & \\
\hline
\end{tabular}

301

302

303

304

305

306

307

308

Table 3: Results of the blind test analysis, after update of the MALDI-TOF MS database, analyzed by Biotyper Software.

\section{Figure legends:}

Figure 1: Reproducible and specific MALDI-TOF MS spectra of Bartonella quintana strain, Bartonella henselae strain, head of fresh Cimex lectularius infected with Bartonella quintana, head of fresh Cimex lectularius infected with Bartonella henselae and control bed bugs analyzed by Flex analysis 3.3 software. a.u., arbitrary units; m/z, mass-to-charge ratio.

Figure 2: The gel view tool of three categories of bed bugs. The circles frame some specific spectra of Cimex lectularius infected with Bartonella quintana (blue), Cimex lectularius infected with Bartonella henselae (green), and Cimex lectularius controls (red).

Figure 3: A: bed bug head MS spectra from infected Cimex lectularius by Bartonella quintana (green dots) and Cimex lectularius controls (red dots) were compared by Principal Component Analysis. B: bed bug head MS spectra from infected Cimex lectularius by 
Bartonella henselae (green dots) and Cimex lectularius controls (red dots) were compared by

317 Principal Component Analysis.

318 Figure 4: Comparison of MALDI-TOF MS profiles of bed bugs infected or not by Bartonella 319 quintana using ClinProTools software. Red and green profiles correspond to average 320 spectrum of control and infected bed bugs, respectively. Peak masses (Da) are indicated 321 above the referred peak. arb.u., arbitrary units; $m / z$, mass-to-charge ratio.

Figure 5: Comparison of MALDI-TOF MS profiles of bed bugs infected or not by Bartonella henselae using ClinProTools software. Red and green profiles correspond to average spectrum of control and infected bed bugs, respectively. Peak masses (Da) are indicated above the referred peak. arb.u., arbitrary units; $m / z$, mass-to-charge ratio.

\section{Supplementary data}

Supplementary data 1: List of all arthropod species included in our in-lab database

\section{Acknowledgements}

We thank Nathalie Duclos (IHU Méditerannée infection, Marseille) for providing us the

Bartonella quintana and Bartonella henselae strains.

The study was funded by the Méditerranée Infection foundation and the National Research Agency under the program "Investissements d'avenir", reference ANR-10-IAHU-03.

\section{Competing interests:}

337 The authors declare that they have no conflicts of interest. 


\section{References}

[1] P. Delaunay, V. Blanc, P. Del Giudice, A. Levy-Bencheton, O. Chosidow, P. Marty, P. Brouqui, Bedbugs and infectious diseases, Clin. Infect. Dis. Off. Publ. Infect. Dis. Soc. Am. 52 (2011) 200-210. doi:10.1093/cid/ciq102.

[2] C. Roberson, Bed Bugs: They Still are Among Us!!, Ala. Nurse. 42 (2015) 11-14; quiz 15.

[3] A.A. Gbakima, B.C. Terry, F. Kanja, S. Kortequee, I. Dukuley, F. Sahr, High prevalence of bedbugs Cimex hemipterus and Cimex lectularis in camps for internally displaced persons in Freetown, Sierra Leone: a pilot humanitarian investigation, West Afr. J. Med. 21 (2002) 268-271.

[4] J. Paul, J. Bates, Is infestation with the common bedbug increasing?, BMJ. 320 (2000) 1141.

[5] H. Leulmi, I. Bitam, J.M. Berenger, H. Lepidi, J.M. Rolain, L. Almeras, D. Raoult, P. Parola, Competence of Cimex lectularius Bed Bugs for the Transmission of Bartonella quintana, the Agent of Trench Fever, PLoS Negl. Trop. Dis. 9 (2015) e0003789. doi:10.1371/journal.pntd.0003789.

[6] B.B. Chomel, R.W. Kasten, K. Floyd-Hawkins, B. Chi, K. Yamamoto, J. RobertsWilson, A.N. Gurfield, R.C. Abbott, N.C. Pedersen, J.E. Koehler, Experimental transmission of Bartonella henselae by the cat flea, J. Clin. Microbiol. 34 (1996) 19521956.

[7] E. Angelakis, C. Socolovschi, D. Raoult, Bartonella quintana in Cimex hemipterus, Rwanda, Am. J. Trop. Med. Hyg. 89 (2013) 986-987. doi:10.4269/ajtmh.13-0182.

[8] D. Cuisance, J. Antoine Rioux, Current status of medical and veterinary entomology in France: endangered discipline or promising science?, Comp. Immunol. Microbiol. Infect. Dis. 27 (2004) 377-392. doi:10.1016/j.cimid.2004.03.007.

[9] A. Yssouf, L. Almeras, D. Raoult, P. Parola, Emerging tools for identification of arthropod vectors, Future Microbiol. 11 (2016) 549-566. doi:10.2217/fmb.16.5.

[10] A. Nebbak, A.C. Willcox, I. Bitam, D. Raoult, P. Parola, L. Almeras, Standardization of sample homogenization for mosquito identification using an innovative proteomic tool based on protein profiling, Proteomics. 16 (2016) 3148-3160. doi:10.1002/pmic.201600287.

[11] F. Tandina, L. Almeras, A.K. Koné, O.K. Doumbo, D. Raoult, P. Parola, Use of MALDI-TOF MS and culturomics to identify mosquitoes and their midgut microbiota, Parasit. Vectors. 9 (2016) 495. doi:10.1186/s13071-016-1776-y.

[12] F. Tandina, S. Niaré, M. Laroche, A.K. Koné, A.Z. Diarra, A. Ongoiba, J.M. Berenger, O.K. Doumbo, D. Raoult, P. Parola, Using MALDI-TOF MS to identify mosquitoes 
collected in Mali and their blood meals, Parasitology. (2018) 1-13. doi:10.1017/S0031182018000070.

[13] A. Yssouf, C. Socolovschi, H. Leulmi, T. Kernif, I. Bitam, G. Audoly, L. Almeras, D. Raoult, P. Parola, Identification of flea species using MALDI-TOF/MS, Comp. Immunol. Microbiol. Infect. Dis. 37 (2014) 153-157. doi:10.1016/j.cimid.2014.05.002.

[14] A. Yssouf, L. Almeras, J.-M. Berenger, M. Laroche, D. Raoult, P. Parola, Identification of tick species and disseminate pathogen using hemolymph by MALDI-TOF MS, Ticks Tick-Borne Dis. 6 (2015) 579-586. doi:10.1016/j.ttbdis.2015.04.013.

[15] A. Fotso Fotso, O. Mediannikov, G. Diatta, L. Almeras, C. Flaudrops, P. Parola, M. Drancourt, MALDI-TOF mass spectrometry detection of pathogens in vectors: the Borrelia crocidurae/Ornithodoros sonrai paradigm, PLoS Negl. Trop. Dis. 8 (2014) e2984. doi:10.1371/journal.pntd.0002984.

[16] A. Yssouf, L. Almeras, J. Terras, C. Socolovschi, D. Raoult, P. Parola, Detection of Rickettsia spp in ticks by MALDI-TOF MS, PLoS Negl. Trop. Dis. 9 (2015) e0003473. doi:10.1371/journal.pntd.0003473.

[17] B. El Hamzaoui, M. Laroche, L. Almeras, J.-M. Bérenger, D. Raoult, P. Parola, Detection of Bartonella spp. in fleas by MALDI-TOF MS, PLoS Negl. Trop. Dis. 12 (2018) e0006189. doi:10.1371/journal.pntd.0006189.

[18] M. Laroche, L. Almeras, E. Pecchi, Y. Bechah, D. Raoult, A. Viola, P. Parola, MALDITOF MS as an innovative tool for detection of Plasmodium parasites in Anopheles mosquitoes, Malar. J. 16 (2017) 5. doi:10.1186/s12936-016-1657-z.

[19] J.M. Sheele, J.F. Anderson, T.D. Tran, Y.A. Teng, P.A. Byers, B.S. Ravi, D.E. Sonenshine, Ivermectin causes Cimex lectularius (bedbug) morbidity and mortality, J. Emerg. Med. 45 (2013) 433-440. doi:10.1016/j.jemermed.2013.05.014.

[20] P. Seng, M. Drancourt, F. Gouriet, B. La Scola, P.-E. Fournier, J.M. Rolain, D. Raoult, Ongoing revolution in bacteriology: routine identification of bacteria by matrix-assisted laser desorption ionization time-of-flight mass spectrometry, Clin. Infect. Dis. Off. Publ. Infect. Dis. Soc. Am. 49 (2009) 543-551. doi:10.1086/600885.

[21] T. Kernif, H. Leulmi, C. Socolovschi, J.-M. Berenger, H. Lepidi, I. Bitam, J.-M. Rolain, D. Raoult, P. Parola, Acquisition and excretion of Bartonella quintana by the cat flea, Ctenocephalides felis felis, Mol. Ecol. 23 (2014) 1204-1212. doi:10.1111/mec.12663.

[22] E. Angelakis, G. Diatta, A. Abdissa, J.-F. Trape, O. Mediannikov, H. Richet, D. Raoult, Altitude-dependent Bartonella quintana genotype $\mathrm{C}$ in head lice, Ethiopia, Emerg. Infect. Dis. 17 (2011) 2357-2359. doi:10.3201/eid1712.110453.

[23] Z. Zeaiter, P.-E. Fournier, D. Raoult, Genomic variation of Bartonella henselae strains detected in lymph nodes of patients with cat scratch disease, J. Clin. Microbiol. 40 (2002) 1023-1030.

[24] A. Nebbak, B. El Hamzaoui, J.-M. Berenger, I. Bitam, D. Raoult, L. Almeras, P. Parola, Comparative analysis of storage conditions and homogenization methods for tick and flea species for identification by MALDI-TOF MS, Med. Vet. Entomol. (2017). doi:10.1111/mve.12250.

[25] A.L. Lawrence, J. Batovska, C.E. Webb, S.E. Lynch, M.J. Blacket, J. Šlapeta, P. Parola, M. Laroche, Accurate identification of Australian mosquitoes using protein profiling, Parasitology. (2018) 1-10. doi:10.1017/S0031182018001658. 


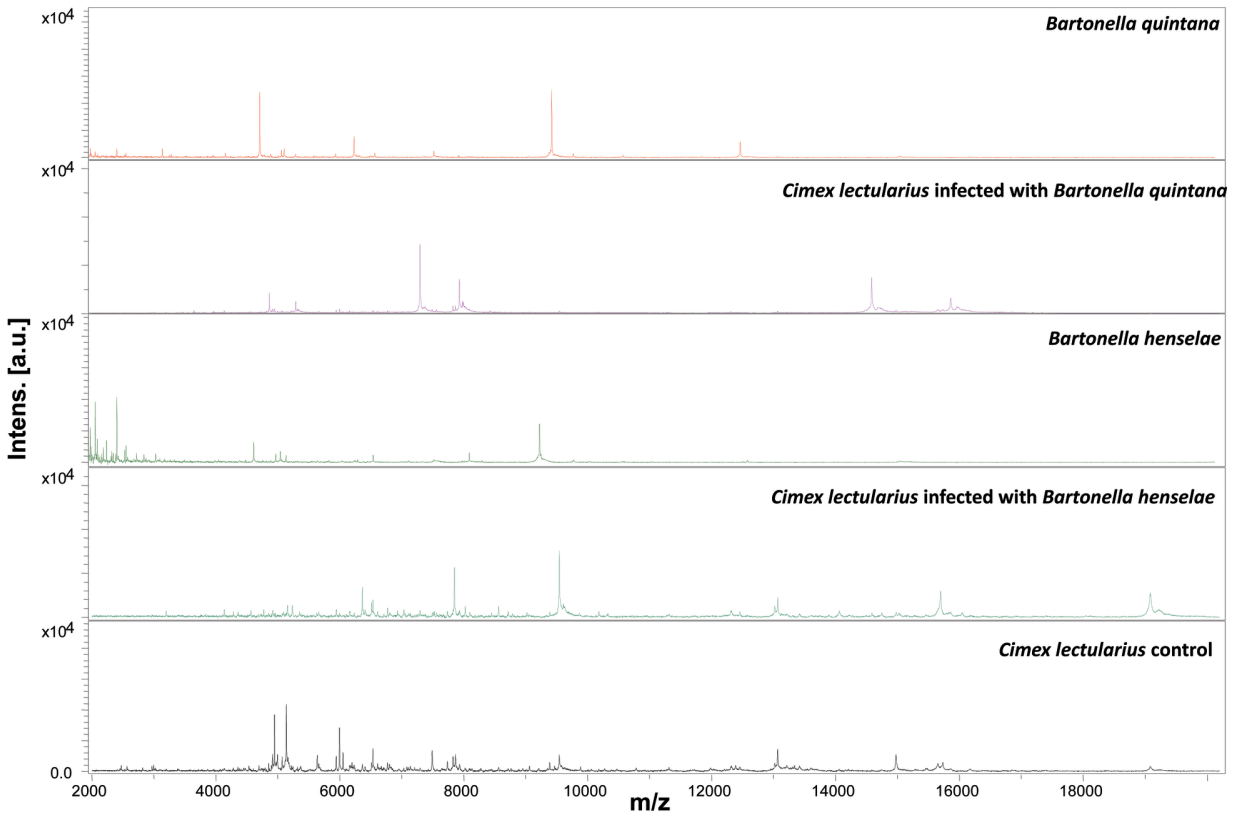


arb. $u$.

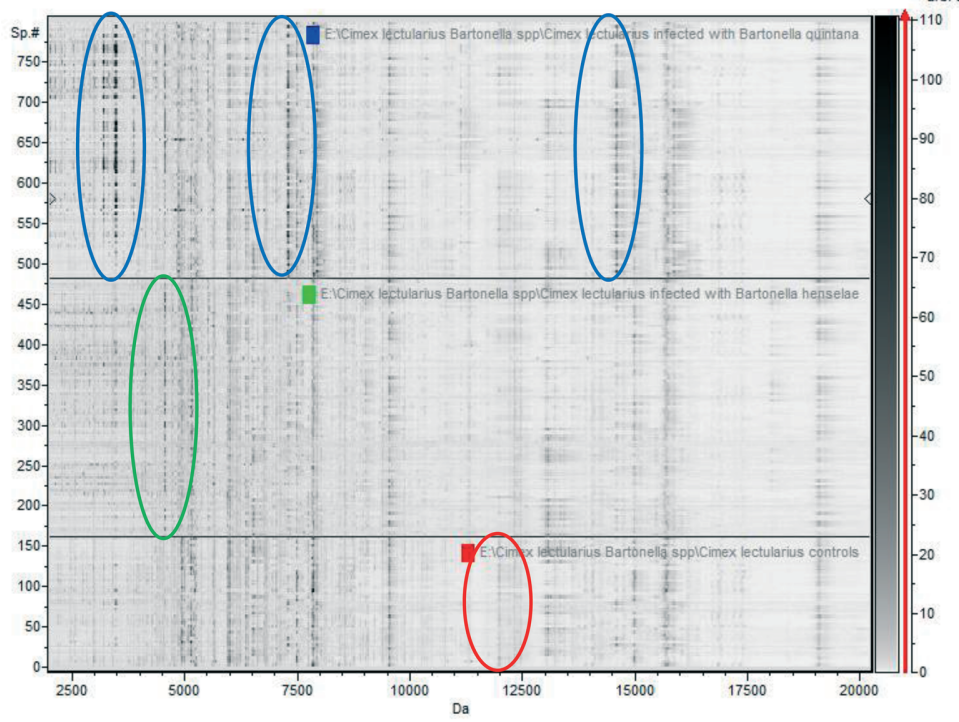

\section{Cimex lectularius infected with \\ Bartonella quintana}

Cimex lectularius infected with Bartonella henselae

\section{Cimex lectularius controls}



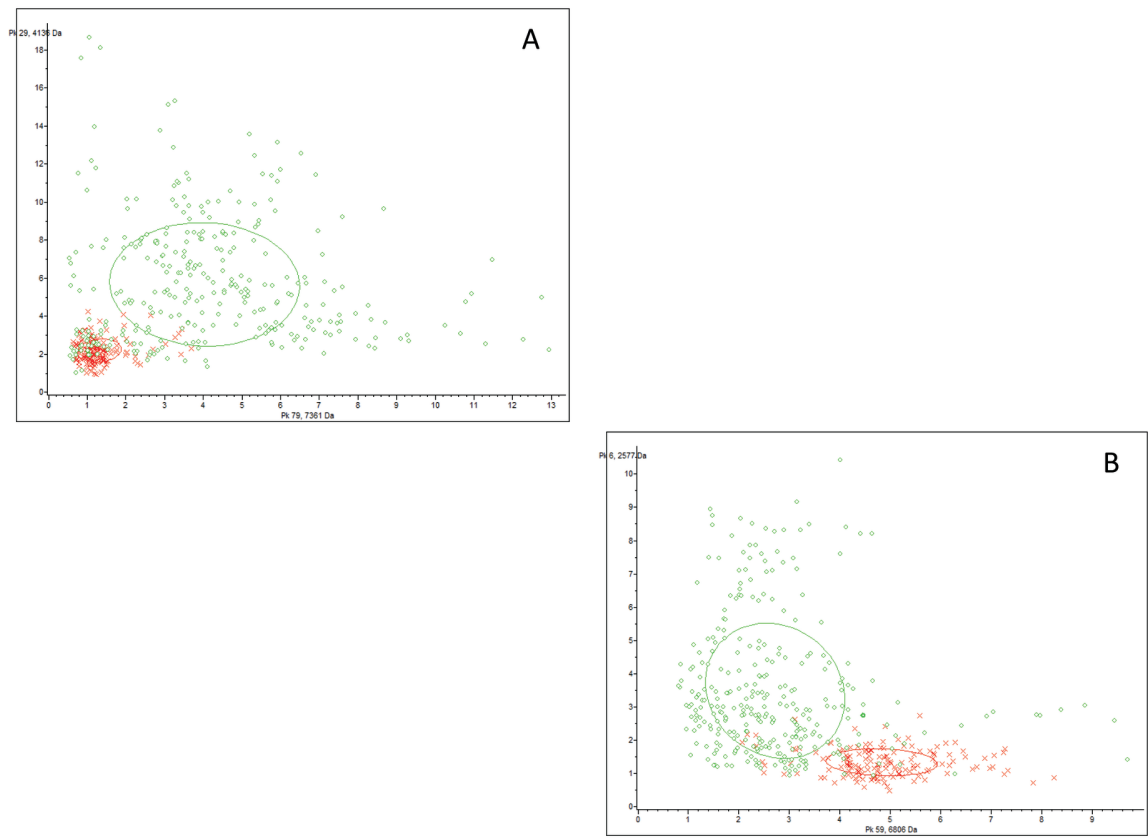

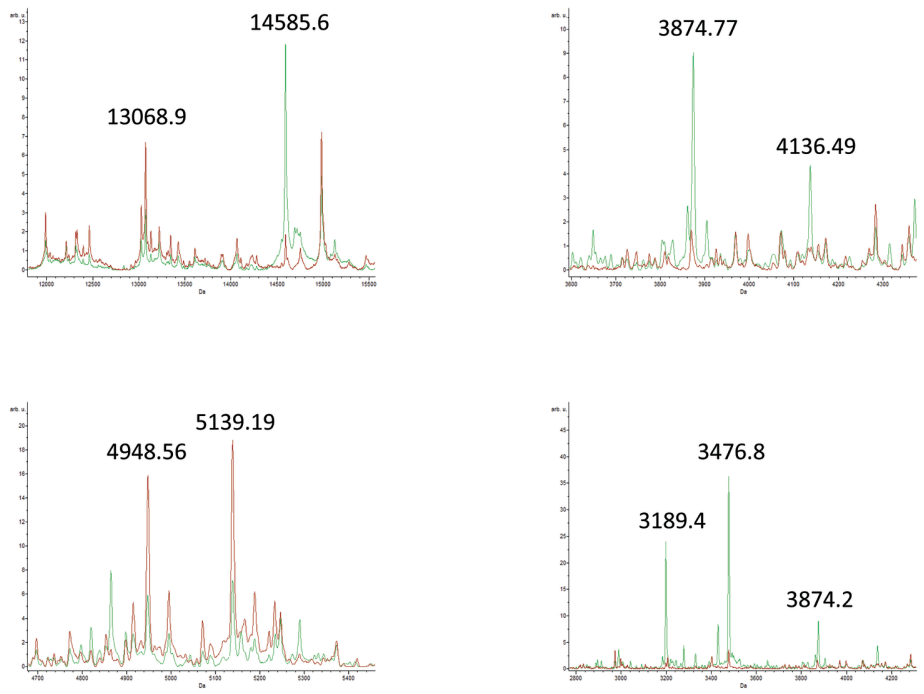

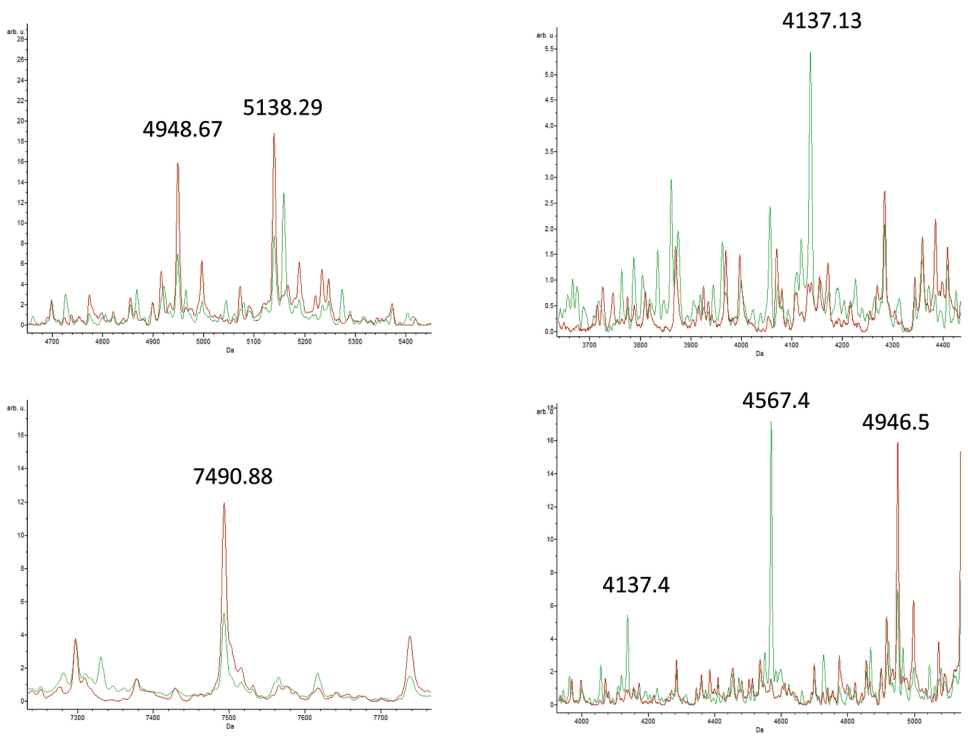